\title{
Optical binding via surface plasmon polariton interference
}

\author{
Natalia Kostina, ${ }^{1}$ Mihail Petrov, ${ }^{1}$ Aliaksandra Ivinskaya, ${ }^{1}$ Sergey Sukhov, ${ }^{2,3}$ Andrey Bogdanov, ${ }^{1}$ Ivan Toftul, ${ }^{1}$ \\ Manuel Nieto-Vesperinas, ${ }^{4}$ Pavel Ginzburg, ${ }^{1,5}$ and Alexander Shalin ${ }^{1,6}$ \\ ${ }^{1}$ ITMO University, Department of Nanophotonics and Metamaterials, Saint-Petersburg, 199034, Russia \\ ${ }^{2}$ CREOL, University of Central Florida, Orlando, Florida, 32816-2700, USA \\ ${ }^{3}$ Kotelnikov Institute of Radio Engineering and Electronics of Russian Academy of Science (Ulyanovsk Branch), Ulyanovsk, 432071, Russia \\ ${ }^{4}$ Instituto de Ciencia de Materiales de Madrid, Consejo Superior de Investigaciones Cientificas, \\ Campus de Cantoblanco, Madrid E-28049, Spain \\ ${ }^{5}$ School of Electrical Engineering, Tel Aviv University, Tel Aviv, 6997801, Israel \\ ${ }^{6}$ Ulyanovsk State University, Ulyanovsk, 432017, Russia
}

(Received 29 May 2018; revised manuscript received 27 December 2018; published 13 March 2019)

\begin{abstract}
Optical binding allows creation of mechanically stable nanoparticle configurations owing to formation of self-consistent optical trapping potentials. While the classical diffraction limit prevents achieving deeply subwavelength arrangements, auxiliary nanostructures enable tailoring optical forces via additional interaction channels. Here, a dimer configuration next to a metal surface was analyzed in detail and the contribution of surface plasmon polariton waves was found to govern the interaction dynamics. It is shown that the interaction channel, mediated by resonant surface waves, enables achieving subwavelength stable dimers. Furthermore, the vectorial structure of surface modes allows binding between two dipole nanoparticles along the direction of their dipole moments, contrary to vacuum binding, where a stable configuration is formed in the direction perpendicular to the polarization of the dipole moments. In addition, the enhancement by one order of magnitude of the optical binding stiffness is predicted owing to the surface plasmon polariton interaction channel. These phenomena pave the way for developing new flexible optical manipulators, allowing for control over a nanoparticle trajectory on subwavelength scales and opening opportunities for optical-induced anisotropic (i.e., with different periods along the field polarization as well as perpendicular to it) organization of particles on a plasmonic substrate.
\end{abstract}

DOI: 10.1103/PhysRevB.99.125416

\section{INTRODUCTION}

Light carries momentum which can influence matter through optical forces, enabling manipulation of micro- and nanoscale objects [1] and even atom ensembles [2]. The methods of optical tweezing $[3,4]$ rely on attraction of small objects to regions of high field intensity. Spatially nonuniform intensity distributions used for positioning microobjects at a predefined pattern can be achieved with a nanostructured environment or by interference of several beams. Yet, since the early years of optical tweezing experiments, it has been discovered that several particles tend to self-organize under homogeneous illumination $[5,6]$. This effect is referred to as transverse optical binding. The interference between incident and scattered light, owing to its interaction with particles, results in the formation of a set of potential wells defining stable positions of particles. Optical binding has been intensively studied both theoretically [7-11] and experimentally [12-16], including as a prospective method for self-organization of particles. However, the strength of optical binding drops rapidly with nanoparticle size as the scattering is proportional to the nanoparticle radius $R$ as $\sim R^{6}$. On the other hand, the viscous damping is also reduced for smaller particles, which makes the fluctuations and stochastic processes in liquids to be more influential. As a result, for reliable optical control of subwavelength nanoparticles, strong optical fields are required. In order to achieve this with available laser intensities, the optical binding can be enhanced by localized plasmon resonances of the nanoparticles $[8,17,18]$. The localized plasmons can improve trapping efficiency at hot spots of a corrugated metal surface $[19,20]$ or provide particle acceleration against beam direction in plasmonic V-grooves [21]. Threedimensional structures of plasmonic particles or alternating metal-dielectric layers, such as metamaterials [22-24], can be also employed to trap or manipulate nanoparticles, e.g., for realization of optical pulling forces attracting nanoparticles to a light source $[25,26]$. In the context of optical binding, flat metal surfaces may be also very relevant. The excitation of propagating surface plasmon polartions (SPPs) and induced optical thermal forces are responsible for self-organization of micron-sized nanoparticles [27]. Moreover, the direct momentum transfer from SPP to micron-sized particles [28,29] can be used for enhancing the optical forces near planar metallic surfaces, which can be used for sorting and ordering of nanoparticles [15,30,31]. Recently, it was suggested that SPP modes can open the way for manipulating the optical forces acting on nanosized particles by the directional excitation of the propagating SPP modes [32-37].

Here, we propose another mechanism of transverse optical binding via excitation of SPP modes (SPP binding) near a metallic planar interface. This mechanism is based on far-field interaction through the interference of SPP waves 
and it is different to the formation of resonant nanoparticle molecules due to their near-field interaction $[17,18,38,39]$. Compared to common transverse binding in a free space (photon binding) [40], the proposed approach has several advantages: (i) It can enhance the binding effect for small nanoparticles due to the resonant excitation of SPP modes; (ii) the distance between the bound pair of nanoparticles is defined by the SPP effective wavelength and, thus, can be significantly smaller, surpassing the diffraction limit; and (iii) the binding occurs in the direction of dipole polarization in accordance with the directivity of SPP emission, which differs from the case of a free space binding, where stable configurations are formed in the direction perpendicular to the dipole moments. In this paper, we theoretically show how SPP-based transverse optical binding can bring new features.

\section{MODEL AND MAIN EQUATIONS}

We consider two identical nanoparticles placed close to a planar metallic interface at coordinates $\mathbf{r}_{1}$ and $\mathbf{r}_{2}$ in the field of a normally incident plane wave (see Fig. 1). We assume that the nanoparticles have radius $R$ and are made of a dielectric material with permittivity $\varepsilon$. In the dipole approximation, the radius of the nanoparticles $R$ is much smaller than the typical scale of the electric field variations. In this limit, the timeaveraged optical force acting on a nanoparticle is given by the expression [41]

$$
\mathbf{F}=\frac{1}{2} \operatorname{Re} \sum_{i} p_{i}^{*} \nabla E_{i}(\mathbf{r}, \omega)
$$

where $E_{i}(\mathbf{r}, \omega)$ is the $i$ th component of a local field.

The dipole moment of a nanoparticle $\mathbf{p}(\mathbf{r})$ is defined as $\mathbf{p}(\mathbf{r})=\alpha(\omega) \mathbf{E}(\mathbf{r})$, where $\alpha(\omega)$ is the vacuum dipole polarizability corrected with account for retardation effects:

$$
\frac{1}{\alpha}=\frac{1}{\alpha_{0}}-\frac{i k_{0}^{3}}{6 \pi \varepsilon_{0}}, \quad \alpha_{0}=4 \pi \varepsilon_{0} R^{3} \frac{\varepsilon-\varepsilon_{1}}{\varepsilon+2 \varepsilon_{1}},
$$

where $k_{0}$ is the wave vector in a free space, $\varepsilon_{0}$ is the vacuum permittivity, and $\alpha_{0}$ is the static polarizability, assuming the particle is a sphere. The local electric field includes the

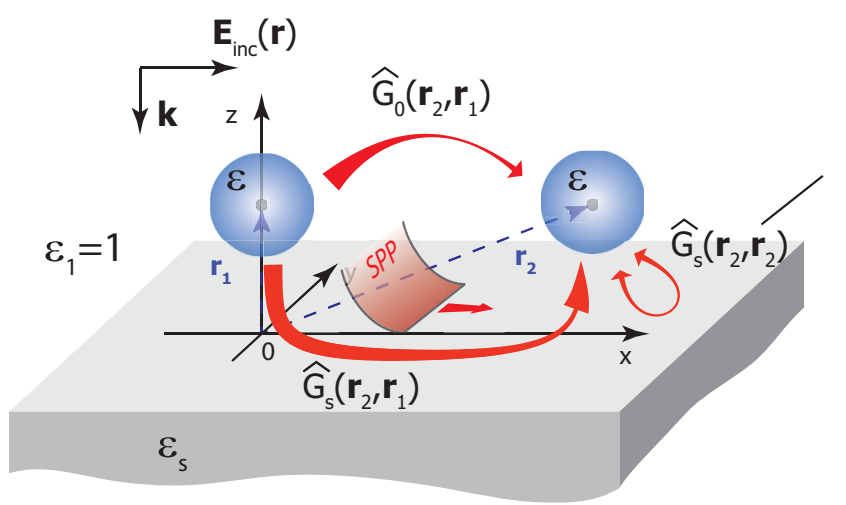

FIG. 1. The scheme of the problem. Nanoparticles with permittivity $\varepsilon$ are positioned at equal distances from a surface $z_{1}=z_{2}=z$. The incident wave is described by the wave vector $\mathbf{k}$ and the electric field $\mathbf{E}_{\text {inc }}(\mathbf{r})$. We assume that the permittivity of the upper half-space is $\varepsilon_{1}=1$ and $\varepsilon_{s}$ is the permittivity of silver substrate. incident plane wave, the multiply rescattered field between particles via free-space and substrate channels, and the selfinduced contribution of each particle through the reflection from the substrate. The local field is given by

$$
\mathbf{E}(\mathbf{r})=\mathbf{E}_{0}(\mathbf{r})+\frac{k_{0}^{2}}{\varepsilon_{0}} \widehat{G}\left(\mathbf{r}, \mathbf{r}_{1}\right) \mathbf{p}_{1}+\frac{k_{0}^{2}}{\varepsilon_{0}} \widehat{G}\left(\mathbf{r}, \mathbf{r}_{2}\right) \mathbf{p}_{2} .
$$

Here, the first term on the right-hand side $\mathbf{E}_{0}$ is the amplitude of the external plane wave $\mathbf{E}_{\text {inc }}(\mathbf{r})$ with the term corresponding to the reflection from the metallic substrate taken into account, while the second and third terms correspond to the field generated by the first and second nanoparticles, respectively. The total Green's function $\widehat{G}\left(\mathbf{r}, \mathbf{r}_{0}\right)=$ $\widehat{G}_{0}\left(\mathbf{r}, \mathbf{r}_{0}\right)+\widehat{G}_{s}\left(\mathbf{r}, \mathbf{r}_{0}\right)$ is a sum of the scattered $\widehat{G}_{s}$ and vacuum $\widehat{G}_{0}$ components, respectively [43]. The sum of terms on the right-hand side of Eq. (3) are the fields of the two excited dipoles, resulting in the formation of the interference pattern. The polarizability tensor can be simplified with respect to the self-action Green's function component $\widehat{G}_{s}\left(\mathbf{r}_{i}, \mathbf{r}_{i}\right)$ :

$$
\mathbf{p}_{i}=\widehat{\alpha}_{i, \mathrm{eff}}^{s}\left[\mathbf{E}_{0}\left(\mathbf{r}_{i}\right)+\frac{k_{0}^{2}}{\varepsilon_{0}} \widehat{G}\left(\mathbf{r}_{i}, \mathbf{r}_{j}\right) \mathbf{p}_{j}\right], \quad i=1,2, j=2,1 .
$$

Here, we have introduced the effective polarizability tensor $\widehat{\alpha}_{i, \mathrm{eff}}^{s}$ as follows:

$$
\begin{aligned}
\widehat{\alpha}_{i, \mathrm{eff}}^{s}\left(\mathbf{r}_{i}, \omega\right)= & \alpha(\omega)\left[\widehat{I}-\alpha(\omega) \frac{k_{0}^{2}}{\varepsilon_{0}} \widehat{G}_{s}\left(\mathbf{r}_{i}, \mathbf{r}_{i}, \omega\right)\right]^{-1}, \\
& i=1,2,
\end{aligned}
$$

if $\widehat{I}$ corresponds to the unitary dyad. This tensor gives a correction of a vacuum polarizability $\alpha(\omega)$ accounting for the nanoparticle self-action through the substrate $[33,43]$. This tensor is diagonal, as is $\widehat{G}_{s}\left(\mathbf{r}_{i}, \mathbf{r}_{i}\right)$ in the case of a flat isotropic substrate [43].

Without loss of generality, we fix the position of the first particle in the origin of the coordinate system at $x_{1}=0$ and $y_{1}=0$ and will consider the force acting on the second particle only. Computing the field at the point of the dipole according to the expression in Eq. (3), one can achieve a system of equations for the dipole moments $\mathbf{p}_{1}$ and $\mathbf{p}_{2}$ (see Appendix A), and, in the special case of normal incidence of the plane wave, the expression for the dipole moments can be simplified even further:

$$
\begin{gathered}
\mathbf{p}_{i}=\widehat{\alpha}_{i, \mathrm{eff}}^{R} \mathbf{E}_{0}\left(\mathbf{r}_{i}\right), \\
\widehat{\alpha}_{i, \mathrm{eff}}^{R}\left(\mathbf{r}_{i}, \omega\right)=\alpha(\omega)\left[\widehat{I}-\alpha(\omega) \frac{k^{2}}{\varepsilon_{0}} \widehat{G}_{s}\left(\mathbf{r}_{i}, \mathbf{r}_{i}, \omega\right)\right. \\
\left.-\frac{k^{4}}{\varepsilon_{0}^{2}} \alpha(\omega) \widehat{G}\left(\mathbf{r}_{i}, \mathbf{r}_{j}\right) \widehat{\alpha}_{j, \mathrm{eff}}^{s}\left(\mathbf{r}_{j}, \omega\right) \widehat{G}\left(\mathbf{r}_{j}, \mathbf{r}_{i}\right)\right]^{-1} \\
\times\left[\widehat{I}+\frac{k^{2}}{\varepsilon_{0}} \widehat{G}\left(\mathbf{r}_{i}, \mathbf{r}_{j}\right) \widehat{\alpha}_{j, \text { eff }}^{s}\left(\mathbf{r}_{j}, \omega\right)\right], \\
i=1,2 \quad j=2,1 .
\end{gathered}
$$

Now, the polarizability $\widehat{\alpha}_{\text {eff }}^{R}$ (see Appendix A for the details) includes all the interaction channels: (i) the self-action of the nanoparticles through the substrate and (ii) the crossaction of the two nanoparticles via the vacuum and the 


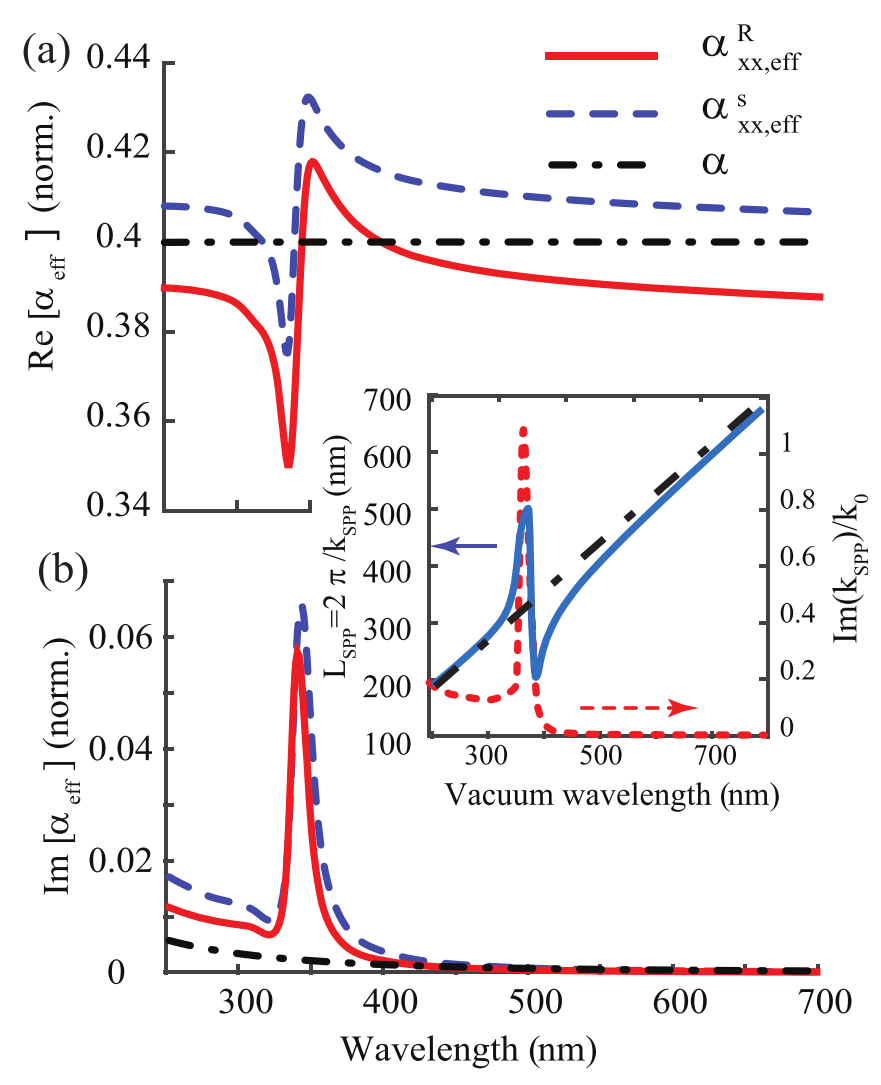

FIG. 2. The spectrum of the real (a) and imaginary (b) parts of the $x x$ components of the effective polarizability tensor $\hat{\alpha}_{\text {eff }}^{s}$ (blue dashed line) and $\hat{\alpha}_{\text {eff }}^{R}$ (red solid line) shown along with the vacuum polarizability $\alpha$ (black dash-dotted line). The polarizability is normalized over $4 \pi \varepsilon_{0} R^{3}$ and calculated for a spherical nanoparticle of radius $R=15 \mathrm{~nm}$ with $\varepsilon=3$ whose center is located above the surface at $z=20 \mathrm{~nm}$. The inset shows the dependence on the vacuum wavelength of the SPP typical period, denoted as $L_{\mathrm{SPP}}=2 \pi / k_{\mathrm{SPP}}$ (upper $x$ axis), and the imaginary part of the SPP wave vector (lower $x$ axis). The dielectric permittivity of the silver substrate $\varepsilon_{s}$ was taken from Johnson and Christy [42].

substrate. Moreover, it is worth mentioning that though the effective polarizability tensor $\widehat{\alpha}_{\text {eff }}^{s}$ is diagonal, the tensor $\widehat{\alpha}_{\text {eff }}^{R}$ is nondiagonal, as the presence of the second nanoparticle does not preserve translational symmetry of the system.

The excitation of SPP modes affects both the effective polarizability due to the substrate mediated self-action and the cross action of the nanoparticles. The spectra of the real and imaginary parts of the $x x$ components of $\widehat{\alpha}_{\text {eff }}^{R}$ (solid line) and $\widehat{\alpha}_{\text {eff }}^{s}$ (dashed line) are plotted in Figs. 2(a) and 2(b), respectively, for the case of a silver substrate. The vacuum polarizability $\alpha$ is also shown in this figure with a dash-dotted line. We observe that the effective polarizabilities have a resonance at around $350 \mathrm{~nm}$. From the inset of Fig. 2, one can see that this wavelength corresponds to the SPP resonant excitation for the silver/vacuum interface, which is defined by the condition $\operatorname{Re}\left[\varepsilon_{s}(\omega)\right]+1=0$, and also corresponds to the maximal value of the real part of the SPP wavevector $k_{S P P}=k_{0} \sqrt{\varepsilon_{s} /\left(\varepsilon_{s}+1\right)}$, where $\varepsilon_{s}$ is the permittivity of the silver substrate [42]. In the inset, the effective wavelength of the SPP mode defined as $L_{S P P}=2 \pi / \operatorname{Re}\left(k_{S P P}\right)$ is also shown.
The strong enhancement of the imaginary part of the effective polarizability is a sign of strong rescattering of light into the SPP mode.

\section{RESULTS AND DISCUSSION}

\section{A. Binding via SPP}

By determining the dipole moments of nanoparticles, one can calculate the optical force acting on each nanoparticle using Eq. (1) (see the details in Appendix A). In the following, we will refer to the optical force acting on the second nanoparticle only, fixing the first nanoparticle in the coordinate origin. In order to find the equilibrium positions of the nanoparticle, we plot the dependence of the $x$ component of the optical force as a function of interparticle distance along the $x$ axis as shown in Fig. 3(a). The force is normalized to the optical pressure force acting on the same nanoparticle in vacuum $F_{0}=1 / 2 k\left|E_{0}\right|^{2} \operatorname{Im}[\alpha(\omega)]$, where $\left|E_{0}\right|$ is the amplitude of the incident plane wave, and the particles' parameters are the same throughout the whole paper: radius $R=15 \mathrm{~nm}$ and dielectric permittivity $\varepsilon=3$. One can see that the force periodically varies with coordinate, which is due to the interference pattern formed in the vicinity of the metal surface, and the zero-force points show equilibrium positions. These points can be stable along $x$ if the force is restoring (shown with solid circles, i.e., point 1) and unstable otherwise (shown with white filled circles). One should also note that when the nanoparticles approach each other, the force goes to minus infinity until the nanoparticles touch each other. However, this case is out of the scope of the present paper.

To identify the role of plasmons in the interaction force, we have excluded the SPP contribution from the Green's function by integrating over the free space modes only (transverse component of the wave vector is limited by the incident wave vector $\mathbf{k}, k_{r}<k_{1}$ ) in the spectral representation (see Appendix B). One can see that in the absence of SPPs the interaction force becomes one order of magnitude weaker, and the period between stable positions is significantly enlarged, being defined by the vacuum wavelength of photons. Moreover, the equilibrium points shown with blue circles are stable both along the $x$ and $y$ directions, making them globally stable, which does not happen for equilibrium positions along the $y$ coordinate in the case of binding with solely photons. To illustrate this, we plotted the $F_{y}$ force-see Fig. 3(b) - as a function of the transverse angle $\phi$ (see the inset in Fig. 3) in the vicinity of points of stable equilibrium. One can see in Fig. 3(c) that the dependence of the binding length on the vacuum wavelength and the period of the SPP wave, which is equal to $L_{\mathrm{SPP}}$. One can see that the binding distance is fully defined by the period of the SPP wave when the excitation condition is fulfilled, thus providing the binding at distances significantly shorter than the vacuum wavelength. This also strongly differs from work of Salary et al. [17], where the optical forces between two nanoparticles over a metallic substrate were considered in the regime, where the interaction force is mainly defined by the near-field components.

In order to support the results discussed above, we have performed numerical simulations with the COMSOL MULTYPHYSICS package [see Fig. 3(d), scatter line]; numerical simulation shows good correspondence with the solution 

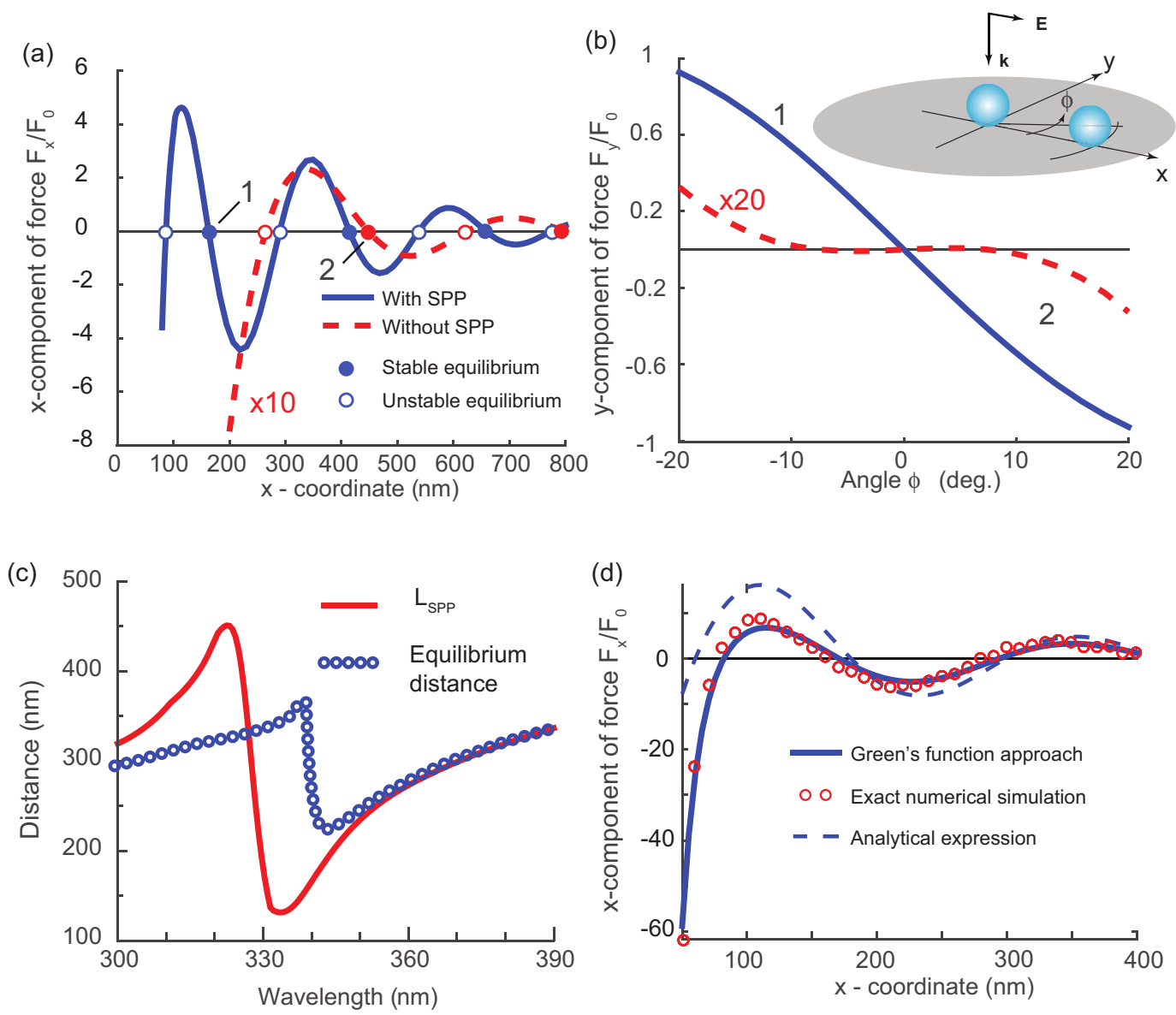

FIG. 3. (a) The $x$ component of optical force plotted along the $x$ axis coinciding with the direction of electric field polarization. The blue solid line denotes the force with account for all interaction channels. The red dashed line is for the interaction through the free space photons only. (b) The $y$ component of the optical force in the direction perpendicular to the $x$ axis, showing the stability of the binding position in the direction transverse to the $x$ axis. The blue solid line and red dashed line correspond to the force calculated with or without account for the SPP interaction channel for the equilibrium positions labeled 1 and 2 in panel (a), respectively. The results are shown for the wavelength $\lambda=350 \mathrm{~nm}$. The values of the force for the red dashed lines are multiplied by factors of 10 and 20 in panels (a) and (b), respectively. (c) The distance between the stable equilibrium positions obtained from panel (a) compared with the distance $L=2 \pi / k_{\mathrm{SPP}}$. (d) Comparison of the optical force calculated within Green's function approach as in Fig. 3(a) and calculated numerically with COMSOL MULTYPHYSICS package. The approximate analytical expression given by Eq. (7) for the SPP-induced force is also shown by the dashed line. All the results shown in the figure are computed for $R=15 \mathrm{~nm}$ and $z=30 \mathrm{~nm}$.

obtained with Green's function approach [see Fig. 3(d), solid line]. The particles were illuminated by a plane wave, and the numerical integration of the Maxwell stress tensor over a sphere surrounding a particle was performed. The Green's function approach shows good agreement with numerical results also at distances comparable to the nanoparticle size. One can notice that for our range of distances between the nanoparticles the dipole model gives good agreement with numerical simulations. The higher order multipoles do not contribute to the optical force, as evidenced by our calculations in COMSOL MULTYPHYSICS. It is also in good agreement with a paper by Khlebtsov et al. [44]. Moreover, on employing Green's function formalism, we have derived the approximate expression for the contribution of the SPP mode to the optical force (see Appendix $\mathrm{C}$ for the details):

$$
F_{x} \approx \pi\left|p_{x}\right|^{2} \operatorname{Re}\left[\frac{k_{\mathrm{SPP}}^{3} k_{1 z}^{2} k_{2 z}}{k_{0}^{2}\left(1-\varepsilon_{s}\right)} H_{1}^{(1)}\left(k_{\mathrm{SPP}} x\right)\right] \exp \left[-\operatorname{Im}\left(k_{1 z}\right) z\right] .
$$

Here, $k_{1 z}=\sqrt{k_{0}^{2}-k_{\mathrm{SPP}}^{2}}$ and $k_{2 z}=\sqrt{\varepsilon_{s} k_{0}^{2}-k_{\mathrm{SPP}}^{2}}$ are $z$ components of the SPP wave vector in the upper half-space and in the substrate respectively, $H_{1}^{(1)}(q)$ is the first-order Hankel function of the first kind, and $p_{x}$ denotes the $x$ component of the dipole moment of the first or second nanoparticle, as dipole moments for identical nanoparticles are equal. The derived expression illustrates the origin of the SPP mode: the Hankel function describes the SPP mode excited by a dipole and propagating over a flat surface. Its zeros define the equilibrium positions of the nanoparticle. The $z$ component of the wave vector is complex since SPP is a localized wave; thus, the exponent in Eq. (7) shows the decay of the SPPdipole coupling.

It should be stressed that the transverse binding in a vacuum does not provide stable equilibrium positions along the $x$ axis [40]. This difference of SPP and photon binding can be understood through the difference in the scattering diagrams of SPPs and photons. This is illustrated in Fig. 4 where twodimensional maps of the $x$ and $y$ force components are plotted. 

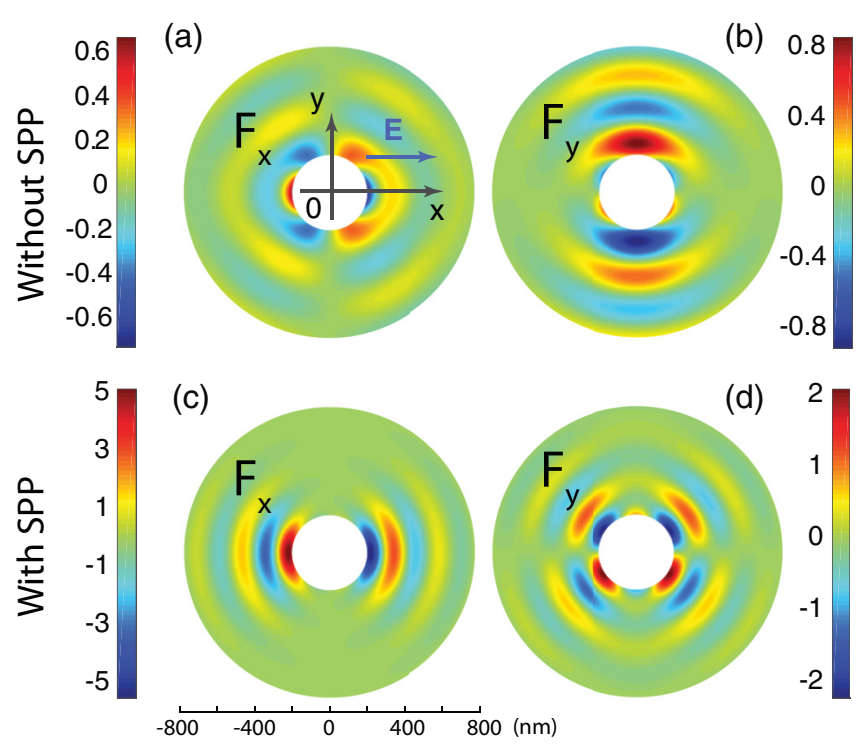

FIG. 4. Two-dimensional maps showing the $x$ and $y$ forces for SPP and photon binding. The different directionality of the scattering pattern is responsible for the different geometry of the stable equilibrium positions. Results are shown for $\lambda=350 \mathrm{~nm}$ and $z=25 \mathrm{~nm}$. The white regions correspond to the high values of the optical force in the vicinity of the first particle, the radius of white regions is around $100 \mathrm{~nm}$; therefore, no equilibrium positions are omitted.

The reshaping of the photon interference [Figs. 4(a) and 4(b)] due to the influence of plasmons [Figs. 4(c) and 4(d)] is clearly seen. The photon binding is well known to have stable configurations perpendicular to the field polarization direction, in accordance with the dipole emission pattern [see Figs. 4(a) and 4(b)]. The SPP binding, on the contrary, has stable configurations along the polarization direction, in which a preferable excitation of SPP modes occurs [see Figs. 4(c) and 4(d)]. It is also worth noting that the amplitudes of the lateral forces are several times higher when SPP modes affect binding.

\section{B. Temporal dynamics of SPP binding}

We illustrate the character of SPP binding by calculating the dynamics of the second nanoparticle motion in the force field of the first nanoparticle, which is fixed at the origin of the coordinates. We consider only two-dimensional motion of the nanoparticle, keeping the $z$ coordinate to be constant. The dynamics is obtained through direct solution of the equations of motion under the external optical force with the account for viscous damping. The details are discussed in Appendix D.

Two typical trajectories are shown in Fig. 5 for two different sets of initial coordinates of the second nanoparticle. The particles are illuminated by a plane wave. The color map shows the intensity of nanoparticle attraction to the equilibrium positions along the $x$ axis, i.e., the amplitude projection of the total force $\mathbf{F}$ on the unit vector $\mathbf{n}$ pointing at the nearest equilibrium position. The arrows show the force field, while the lines show the trajectories with color changing from blue to red as time elapses. One can see that the nanoparticle actively tends to set the position along the $x$ axis where the binding force is the strongest.

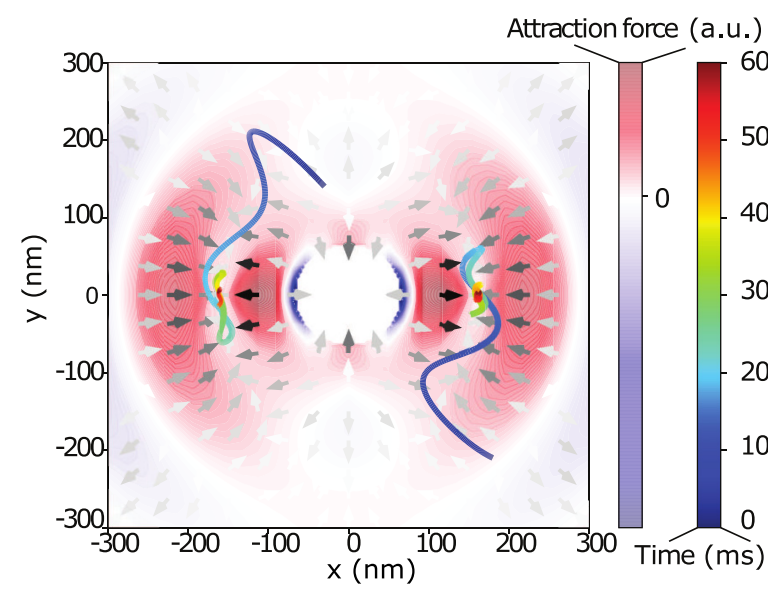

FIG. 5. The dynamics of the second nanoparticle motion. The first nanoparticle is fixed at the origin. The color of the trajectory line denotes the time elapsed since the beginning of motion. The arrows show the force field: darker arrows indicate stronger optical force. The color map at the background shows the force which attracts or repulses the nanoparticle to/from the equilibrium positions at $y=0$ and $x= \pm 175 \mathrm{~nm}$. Color represents the projection of the total force $\mathbf{F}$ on the unit vector $\mathbf{n}$ pointing toward the nearest equilibrium position. The intensity of the red color gives the strength of the nanoparticle attraction, while that of the blue shows repulsion of the nanoparticle. The parameters of computation are $R=15 \mathrm{~nm}, z=25 \mathrm{~nm}$, and $\varepsilon=$ 3. The laser intensity is taken $5 \times 10^{5} \mathrm{~W} / \mathrm{m}^{2}$, and the dimensionless damping factor is $\gamma=0.015$, which corresponds to damping in the vacuum with pressure $10^{-6}$ atm (see Appendix D for details of the simulation method).

\section{Stiffness of SPP binding}

The important parameter, which characterizes the stability of the equilibrium states, is the stiffness of the trap. At the equilibrium positions, the total optical force is zero, but when shifted from the stable positions the nanoparticles undergo action of a restoring force, which is locally proportional to the amplitude of the displacement $F_{x}=-\kappa_{x} \Delta x$, with the parameter $\kappa_{x}$ characterizing the stiffness of the system along the $x$ direction. However, this approximation of the restoring force applies only to the gradient component of the optical force. Indeed, we consider the nanoparticles significantly smaller than the wavelength, which results in low and nonresonant polarizabilities so that where $\operatorname{Im}\left(\alpha_{\text {eff }}\right) \ll \operatorname{Re}\left(\alpha_{\text {eff }}\right)$, as $\operatorname{Im}\left(\alpha_{\text {eff }}\right) \simeq\left(R^{6} / \lambda^{3}\right)$, and $R \ll \lambda$ (see Fig. 2). Thus, the radiation force, which is proportional to the imaginary part of the polarizability, can be neglected (see Appendix D).

The stiffness in the considered system strongly depends on the mechanism of the nanoparticle interaction, and, as can be seen from Fig. 3, it is much higher when the plasmon interaction is enabled. We have plotted (see Fig. 6) the spectral dependence of stiffness parameter $\kappa_{x}$ calculated at the first equilibrium position, labeled by point 1 in Fig. 3(a). To avoid the dependence of the stiffness on the illumination intensity, we have normalized it to the magnitude $\kappa_{0}=F_{0} / R$, which is the stiffness of a system where the vacuum pressure force $F_{0}$ can be restored when the nanoparticle is displaced a distance 


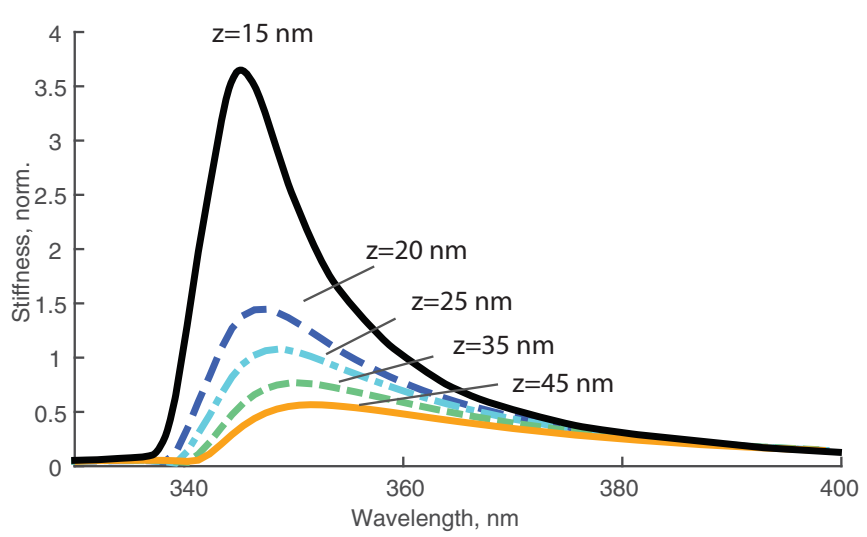

FIG. 6. The stiffness $\kappa_{x}$ of the second equilibrium position in units of $\kappa_{0}=F_{0} / R$ as a function of the excitation wavelength. The spectra are shown for different distances $z$ from the nanoparticle center to the surface. The nanoparticle radius $R=15 \mathrm{~nm}$.

equal to its radius from its equilibrium position. One can see that the stiffness has a strong resonant behavior, which corresponds to the excitation of SPP modes at wavelengths longer than $350 \mathrm{~nm}$. With the increase of the distance from nanoparticle center to the surface the stiffness rapidly drops, as the coupling with the SPP mode decreases.

One can see from Fig. 6 that the spectral maximum of stiffness depends on the height from the surface. This spectral dependence can be better understood by means of Eq. (7). In the case of neglible losses in the substrate, one can get a simple expression for the stiffness at the first stable equilibrium point, (see Appendix C for more details):

$$
\kappa_{x} \approx \pi\left|p_{x}\right|^{2} \frac{\left(k_{\mathrm{SPP}}\right)^{3}\left|k_{1 z}\right|^{2}\left|k_{2 z}\right|}{k_{0}^{2}\left(1-\varepsilon_{s}\right)} Y_{2}\left(q_{1}\right) \exp \left(-2\left|k_{1 z}\right| z\right) .
$$

Here, $Y_{2}(q)$ is the cylindrical Webber function of the second order [45], and $q_{1}$ is the first positive root of $Y_{1}\left(q_{1}\right)=0$, the cylindrical Webber function of the first order. Note that in the regime of SPP excitation without ohmic losses the SPP wave vector can be in a range from $k_{0}$ to $+\infty$ when $1+\varepsilon_{s} \rightarrow 0$. Then the expression given by Eq. (C11) goes to zero in both limiting cases: $\kappa_{x} \rightarrow 0$ as $k_{\mathrm{SPP}} \rightarrow 0 / \infty$, which implies that the stiffness reaches its maximum at some particular wavelength. This wavelength can be defined for each given distance over the substrate $z$. The maximal stiffness can be achieved close to the SPP resonance when the SPP wave vector is $\tilde{k} \approx 6 / z \gg k_{0}$. Then, the maximal stiffness at the $n$th equilibrium position decreases with the distance to the substrate as $z^{-6}$ (see Eq. (C12) in Appendix C):

$$
\kappa_{x, n} \sim\left|p_{x}\right|^{2}\left(\frac{1}{z}\right)^{6} \frac{1}{k_{0}^{2}} Y_{2}\left(q_{2 n+1}\right),
$$

where $q_{2 n+1}$ corresponds to the positive roots of $Y_{1}\left(q_{2 n+1}\right)=0$.

There is a strong dependence of the trap stiffness on the size of the nanoparticles radius and permittivities. With the growth of the particles' radius, the maximal value of the normalized stiffness decreases. The details are given in Appendix F.

\section{Conclusion}

In this work, we consider transverse optical binding based on surface plasmon polariton interference. We show that two nanoparticles placed in the vicinity of a plasmonic interface can form a stable bound dimer with a binding length defined by the SPP wavelength. This allows formation of the dimers with interparticle distance significantly shorter than the free-space wavelength, thus suppressing the diffraction limit. The binding states are formed along the direction of the incident field polarization, in contrast to photon binding, where the stable bound states are formed perpendicular to the polarization direction. The excitation of SPP modes also enhances the amplitude of the binding forces, resulting in a resonant enhancement of the trap stiffness.

\section{ACKNOWLEDGMENTS}

The authors acknowledge the support of the Russian Foundation for Basic Research (Grants No. 16-32-60167, No. 1802-00414, No. 18-29-20063, and No. 18-52-00005). M.I.P. also acknowledges the support from the BASIS Foundation. A.S. acknowledges the support of the Ministry of Education and Science of the Russian Federation (GOSZADANIE, Grant No. 3.4982.2017/6.7). The force calculations were partially supported by Russian Science Foundation (Grant No. 18-72-10127). M.N.-V. is supported by the Spanish MINECO Grants No. FIS2012-36113-C03-03, No. FIS201455563-REDC, and No. FIS2015-69295-C3-1-P.

\section{APPENDIX A: CALCULATION OF A BINDING FORCE}

The force $\mathbf{F}_{2}\left(\mathbf{r}_{2}\right)$ acting on the second nanoparticle is calculated as

$$
\mathbf{F}_{2}\left(\mathbf{r}_{2}\right)=\frac{1}{2} \operatorname{Re}\left[\sum p_{2 i}^{*}\left(\mathbf{r}_{2}\right) \nabla E_{i}\left(\mathbf{r}_{2}\right)\right] .
$$

The introduction of the effective polarizability parameters significantly simplifies the formula for the electric field $\mathbf{E}(\mathbf{r})$ induced by the dipole moments [see Eq. (3)]. By calculating the field amplitude at the points of the dipoles $\mathbf{E}\left(\mathbf{r}_{1}\right)$ and $\mathbf{E}\left(\mathbf{r}_{2}\right)$, one can get a system of linear equations on the dipole moments:

$$
\begin{gathered}
\mathbf{p}_{1}=\widehat{\alpha}_{1, \mathrm{eff}}^{s} \mathbf{E}(\mathbf{r})=\widehat{\alpha}_{1, \mathrm{eff}}^{s}\left[\mathbf{E}_{0}\left(\mathbf{r}_{1}\right)+\frac{k_{0}^{2}}{\varepsilon_{0}} \widehat{G}\left(\mathbf{r}_{1}, \mathbf{r}_{2}\right) \mathbf{p}_{2}\right] \\
\mathbf{p}_{2}=\widehat{\alpha}_{2, \mathrm{eff}}^{s}\left[\mathbf{E}_{0}\left(\mathbf{r}_{2}\right)+\frac{k_{0}^{2}}{\varepsilon_{0}} \widehat{G}\left(\mathbf{r}_{2}, \mathbf{r}_{1}\right) \mathbf{p}_{1}\right] \\
\widehat{\alpha}_{i, \mathrm{eff}}^{s}\left(\mathbf{r}_{i}, \omega\right)=\alpha(\omega)\left[\widehat{I}-\alpha(\omega) \frac{k_{0}^{2}}{\varepsilon_{0}} \widehat{G}_{s}\left(\mathbf{r}_{i}, \mathbf{r}_{i}, \omega\right)\right]^{-1} \\
i=1,2
\end{gathered}
$$

Solving this system, one may get

$$
\begin{aligned}
\mathbf{p}_{1}= & \widehat{\alpha}_{1, \mathrm{eff}}^{s}\left(\mathbf{E}_{0}\left(\mathbf{r}_{1}\right)+\frac{k_{0}^{2}}{\varepsilon_{0}} \widehat{G}\left(\mathbf{r}_{1}, \mathbf{r}_{2}\right)\right. \\
& \left.\times\left\{\widehat{\alpha}_{2, \mathrm{eff}}^{s}\left[\mathbf{E}_{0}\left(\mathbf{r}_{2}\right)+\frac{k_{0}^{2}}{\varepsilon_{0}} \widehat{G}\left(\mathbf{r}_{2}, \mathbf{r}_{1}\right) \mathbf{p}_{1}\right]\right\}\right),
\end{aligned}
$$




$$
\begin{aligned}
\mathbf{p}_{1}= & \widehat{\alpha}_{1, \mathrm{eff}}^{s}\left[\mathbf{E}_{0}\left(\mathbf{r}_{1}\right)+\frac{k_{0}^{2}}{\varepsilon_{0}} \widehat{G}\left(\mathbf{r}_{1}, \mathbf{r}_{2}\right) \widehat{\alpha}_{2, \mathrm{eff}}^{s} \mathbf{E}_{0}\left(\mathbf{r}_{2}\right)\right] \\
& +\frac{k_{0}^{4}}{\varepsilon_{0}^{2}} \widehat{\alpha}_{1, \mathrm{eff}}^{s} \widehat{G}\left(\mathbf{r}_{1}, \mathbf{r}_{2}\right) \widehat{\alpha}_{2, \mathrm{eff}}^{s} \widehat{G}\left(\mathbf{r}_{2}, \mathbf{r}_{1}\right) \mathbf{p}_{1},
\end{aligned}
$$

The last expression in Eq. (A5) can be simplified even further, if one renormalizes the effective polarizability tensor with account for nanoparticle cross action:

$$
\begin{gathered}
\widehat{\alpha}_{i, \mathrm{eff}}^{r}\left(\mathbf{r}_{i}, \omega\right)=\alpha_{i}(\omega)\left[\widehat{I}-\alpha_{i}(\omega) \frac{k^{2}}{\varepsilon_{0}} \widehat{G}_{s}\left(\mathbf{r}_{i}, \mathbf{r}_{i}, \omega\right)\right. \\
\left.-\frac{k^{4}}{\varepsilon_{0}^{2}} \alpha_{i} \widehat{G}\left(\mathbf{r}_{i}, \mathbf{r}_{j}\right) \widehat{\alpha}_{j, \mathrm{eff}}^{s} \widehat{G}\left(\mathbf{r}_{j}, \mathbf{r}_{i}\right)\right]^{-1}, \quad i=1,2 \quad j=2,1 .
\end{gathered}
$$

Here, the self-action Green's function $\widehat{G}_{s}\left(\mathbf{r}_{i}, \mathbf{r}_{i}\right)$ contains the scattered part only, whereas the cross-action part $\widehat{G}\left(\mathbf{r}_{i}, \mathbf{r}_{j}\right)=\widehat{G}_{0}\left(\mathbf{r}_{i}, \mathbf{r}_{j}\right)+\widehat{G}_{s}\left(\mathbf{r}_{i}, \mathbf{r}_{j}\right)$ includes both vacuum and scattered parts, determining the cross interaction through vacuum and via substrate respectively. The final expression for the dipole moment will be as follows:

$$
\begin{gathered}
\mathbf{p}_{i}=\widehat{\alpha}_{i, \mathrm{eff}}^{r}\left[\mathbf{E}_{0}\left(\mathbf{r}_{i}\right)+\frac{k^{2}}{\varepsilon_{0}} \widehat{G}\left(\mathbf{r}_{i}, \mathbf{r}_{j}\right) \widehat{\alpha}_{j, \mathrm{eff}}^{s} \mathbf{E}_{0}\left(\mathbf{r}_{j}\right)\right] \\
i=1,2 j=2,1
\end{gathered}
$$

The case of normal plane-wave incidence on a planar substrate, when the nanoparticles are located at the same height above the surface (see Fig. 1 in the main text) is of a particular interest. In this case, the external electric field $\mathbf{E}_{0}$ is equal in the centers of both nanoparticles, and thus the dipole moment has a very simple form:

$$
\begin{gathered}
\mathbf{p}_{i}=\widehat{\alpha}_{i, \mathrm{eff}}^{R} \mathbf{E}_{0}\left(\mathbf{r}_{i}\right), \\
\widehat{\alpha}_{i, \mathrm{eff}}^{R}=\widehat{\alpha}_{i, \mathrm{eff}}^{r}\left[\widehat{I}+\frac{k^{2}}{\varepsilon_{0}} \widehat{G}\left(\mathbf{r}_{i}, \mathbf{r}_{j}\right) \widehat{\alpha}_{j, \mathrm{eff}}^{s}\right], \quad i=1,2 \quad j=2,1 .
\end{gathered}
$$

The optical force component, then, can be calculated as

$$
\begin{aligned}
F_{2 x}\left(\mathbf{r}_{2}\right)= & \frac{1}{2} \operatorname{Re}\left[\sum_{n=x, y, z} p_{2 n}^{*}\left(\mathbf{r}_{2}\right) \partial_{x_{2}} E_{n}\left(\mathbf{r}_{2}\right)\right] \\
= & \frac{1}{2} \operatorname{Re}\left\{\sum _ { n = x , y , z } p _ { 2 n } ^ { * } ( \mathbf { r } _ { 2 } ) \left[\partial_{x_{2}} E_{0 n}\left(\mathbf{r}_{2}\right)\right.\right. \\
& +\frac{k_{0}^{2}}{\varepsilon_{0}} \sum_{m=x, y, z} \partial_{x_{2}^{\prime}} G_{s, n m}\left(\mathbf{r}_{2}^{\prime}, \mathbf{r}_{2}\right) p_{2 m} \\
& \left.\left.+\frac{k_{0}^{2}}{\varepsilon_{0}} \sum_{m=x, y, z} \partial_{x_{2}} G_{n m}\left(\mathbf{r}_{2}, \mathbf{r}_{1}\right) p_{1 m}\right]\right\} .
\end{aligned}
$$

The $y$ and $z$ components can be calculated with the same expression (A11) by substituting the partial derivative with $\partial_{y}$ and $\partial_{z}$ respectively.

\section{APPENDIX B: GREEN'S FUNCTION}

The Green's function tensor of two half-spaces with permittivities $\varepsilon_{1}$ (for $z>0$ ) and $\varepsilon_{2}$ (for $z \leqslant 0$ ) can be expressed in cylindrical coordinates through [43] (for $z>0$ ):

$$
\widehat{G}(\rho, \varphi, z>0)=\frac{i k_{1}}{8 \pi^{2}} \int_{0}^{\infty} \widehat{M}(s, \rho, \varphi) \exp \left(2 i s_{z 1} \tilde{z}\right) d s,
$$

where $k_{1}$ is the wave vector in the upper space, $s=k_{r} / k_{0}$ and $s_{z 1}=k_{z 1} / k_{0}$ are the radial and $z$ components of the dimensionless wave vector normalized to the wave vector in the free space, and $\tilde{z}=z k_{0}$ is dimensionless coordinate;

$$
\begin{aligned}
\widehat{M}(s, \rho, \varphi) & =\left(\begin{array}{lll}
m_{x x} & m_{x y} & m_{x z} \\
m_{y x} & m_{y y} & m_{y z} \\
m_{z x} & m_{z y} & m_{z z}
\end{array}\right), \\
m_{x x} & =\frac{s}{s_{z 1}} r_{s} f(s, \rho, \varphi)-s s_{z 1} r_{p} g(s, \rho, \varphi), \\
m_{y y} & =\frac{s}{s_{z 1}} r_{s} g(s, \rho, \varphi)-s s_{z 1} r_{p} f(s, \rho, \varphi), \\
m_{z z} & =2 \pi J_{0}(s \rho) r_{p} \frac{s^{3}}{s_{z 1}} \\
m_{x y} & =m_{y x}=\frac{\left(r_{s}+s_{z 1}^{2} r_{p}\right)}{s s_{z 1}} h(s, \rho, \varphi), \\
m_{x z} & =-m_{z x}=-s r_{p} t(s, \rho, \varphi), \\
m_{y z} & =-m_{z y}=-s r_{p} w(s, \rho, \varphi),
\end{aligned}
$$

where the functions $f(s, \rho, \varphi), g(s, \rho, \varphi), h(s, \rho, \varphi)$, $t(s, \rho, \varphi), w(s, \rho, \varphi)$ can be expressed:

$$
\begin{aligned}
f(s, \rho, \varphi) & =2 \pi\left[\sin ^{2}(\varphi) J_{0}(s \rho)+\frac{J_{1}(s \rho)}{s \rho} \cos (2 \varphi)\right], \\
g(s, \rho, \varphi) & =2 \pi\left[\cos ^{2}(\varphi) J_{0}(s \rho)-\frac{J_{1}(s \rho)}{s \rho} \cos (2 \varphi)\right], \\
h(s, \rho, \varphi) & =\pi s^{2} J_{2}(s \rho) \sin (2 \varphi), \\
t(s, \rho, \varphi) & =2 \pi i s J_{1}(s \rho) \cos (\varphi), \\
w(s, \rho, \varphi) & =2 \pi i s J_{1}(s \rho) \sin (\varphi) .
\end{aligned}
$$

Here, $J_{n}(z)$ is the first kind Bessel function of the order $n$.

\section{APPENDIX C: ANALYTICAL EXPRESSION}

Here, we analyze the $x$ component of the optical force acting on the nanoparticles when normal incident light is polarized along the $x$ axis. This is the case considered in Fig. 3. According to Eq. (1), the expression for the force will be as follows:

$$
F_{x}=\frac{1}{2} \operatorname{Re}\left(\sum_{i} p_{i}(\mathbf{r})^{*} \partial_{x} E_{i}\left(\mathbf{r}_{i}\right)\right) .
$$

In order to get a simple analytical result showing all the key features of the SPP-assisted force, we will take into account that the effective $\hat{\alpha}^{R}$ tensor has diagonal domination, which implies that $\alpha_{i i}^{R} \gg \alpha_{i j}^{R}, \quad i \neq j$.

The expression in Eq. (C1) can be simplified:

$$
F_{x}=\frac{1}{2} \operatorname{Re}\left(p_{x}^{*} \partial_{x} E_{x}^{s}\right) .
$$


The electrical field generated by the dipole at the point with radius vector $\mathbf{r}$ can be expressed through the Green's function $E_{x}^{s}(\mathbf{r})=4 \pi k_{0}^{2} G_{s, x x}(\mathbf{r},(0,0, z)) p_{x}$.

Then we have an expression for the lateral component of the optical force written in a very simple form:

$$
F_{x}=2 \pi k_{0}^{2}\left|p_{x}\right|^{2} \operatorname{Re}\left[\partial_{x} G_{s, x x}(\mathbf{r},(0,0, z))\right]
$$

The Green's function is expressed through the integral

$$
\begin{aligned}
G_{s, x x}(x, y, z) & =G_{s, x x}(\rho, \phi, z)=\frac{i k_{1}}{8 \pi^{2}} \int_{0}^{\infty} m_{x x}(\rho, s) \exp \left(2 i s_{1 z} z\right) d s, \quad m_{x x}=s r_{s}(s) \frac{a_{1}(\rho, \phi)}{s_{1 z}}-s s_{1 z} r_{p}(s) a_{2}(\rho, \phi), \\
a_{1} & =2 \pi\left[\sin \left(\phi_{0}\right)^{2} J_{0}(s \rho)+\frac{J_{1}(s \rho)}{s \rho} \cos \left(2 \phi_{0}\right)\right], \quad a_{2}=2 \pi\left[\cos \left(\phi_{0}\right)^{2} J_{0}(s \rho)-\frac{J_{1}(s \rho)}{s \rho} \cos \left(2 \phi_{0}\right)\right], \\
s_{1 z} & =\sqrt{1-s^{2}}, \quad s_{2 z}=\sqrt{\varepsilon_{2}-s^{2}} .
\end{aligned}
$$

Here, we use the same notation as in Appendix B. We are interested only in the component containing $r_{p}$ term as only it gives rise to SPP response, and also we put $\phi=0$. Then,

$$
G_{s, x x}(\rho, \phi, z)=\frac{i k_{1}}{8 \pi^{2}} \int_{0}^{\infty} m_{x x}^{\prime}(\rho, s) \exp \left(2 i s_{1 z} \tilde{z}\right) d s, \quad m_{x x}^{\prime}=-s s_{1 z} r_{p}(s) a_{2}(s, \rho), \quad a_{2}=2 \pi\left[J_{0}(s \rho)-\frac{J_{1}(s \rho)}{s \rho}\right] .
$$

Next, we have

$$
G_{s, x x}(\rho, \phi, z)=-\frac{i k_{1}}{4 \pi} \int_{0}^{\infty} s s_{1 z} r_{p}(s)\left[J_{0}(s \rho)-\frac{J_{1}(s \rho)}{s \rho}\right] \exp \left(2 i s_{1 z} \tilde{z}\right) d s .
$$

With this, we need to compute $\partial_{x} G_{s, x x}$ :

$$
\partial_{x} J_{0}(s \rho)=k_{0} \partial_{\rho} J_{0}(s \rho)=-k_{0} s J_{1}(s \rho), \quad \partial_{x} \frac{J_{1}(s \rho)}{s \rho}=k_{0} s \partial_{s \rho} \frac{J_{1}(s \rho)}{s \rho}=-k_{0} s \frac{J_{2}(s \rho)}{(s \rho)},
$$

which gives us

$$
\partial_{x} G_{s, x x}(\rho, 0, z)=\frac{i k_{1}}{4 \pi} k_{0} \int_{0}^{\infty} s^{2} s_{1 z} r_{p}(s)\left[J_{1}(s \rho)-\frac{J_{2}(s \rho)}{(s \rho)^{2}}\right] \exp \left(2 i s_{1 z} \tilde{z}\right) d s .
$$

In order to compute the integral with the help of complex analysis, we first continue the integral bounds to $-\infty,+\infty$ using the identity

$$
J_{n}(q)=\frac{1}{2}\left[H_{n}^{(1)}(q)-(-1)^{n} H_{n}^{(1)}(-q)\right], \quad \partial_{x} G_{s, x x}(\rho, 0, z)=\frac{i k_{1}}{8 \pi} k_{0} \int_{-\infty}^{\infty} \underbrace{s^{2} s_{1 z} r_{p}(s)\left[H_{1}^{(1)}(s \rho)-\frac{H_{2}^{(1)}(s \rho)}{(s \rho)}\right] \exp \left(2 i s_{1 z} \tilde{z}\right)}_{I(s)} d s .
$$

Now, using Cauchy theorem, we finally evaluate this integral:

$$
\partial_{x} G_{s, x x}(\rho, 0, z)=\left.\frac{i k_{1}}{8 \pi} k_{0} 2 \pi i \operatorname{Res}(I(s))\right|_{s=\tilde{s}}=-\left.\frac{k_{1}}{4} k_{0}(\tilde{s})^{2} \tilde{s}_{1 z}\left[H_{1}^{(1)}(\tilde{s} \rho)-\frac{H_{2}^{(1)}(\tilde{s} \rho)}{(\tilde{s} \rho)}\right] \exp \left(i \tilde{s}_{1 z} \tilde{z}\right) \operatorname{Res}\left(r_{p}(s)\right)\right|_{s=\tilde{s}},
$$

where $\left.\operatorname{Res}\left(r_{p}(s)\right)\right|_{s=\tilde{s}}$ stands for residue, and $\tilde{s}=\sqrt{\varepsilon_{2} \varepsilon_{1} /\left(\varepsilon_{1}+\varepsilon_{2}\right)}$ is dimensionless wave vector of SPP mode.

Finally, computing the explicit expression for the residue and substituting the obtained results into Eq. (C3), one can get

$$
F_{x}=\pi\left|p_{x}\right|^{2} \operatorname{Re}\left\{\frac{(\tilde{k})^{3}\left(\tilde{k}_{1 z}\right)^{2} \tilde{k}_{2 z}}{k_{0}^{2}\left(\varepsilon_{1}-\varepsilon_{2}\right)}\left[H_{1}^{(1)}(\tilde{k} x)-\frac{H_{2}^{(1)}(\tilde{k} x)}{(\tilde{k} x)^{2}}\right] \exp \left(2 i \tilde{k}_{1 z} z\right)\right\} .
$$

Here, we use dimension variables denoting $\tilde{k}=\tilde{s} k_{0}$, and $\tilde{k}_{z 1, z 2}=\tilde{s}_{z 1, z 2} k_{0}$. We can go even further, taking into account that $\left|H_{1}^{(1)}(\tilde{k} x)\right| \ll\left|J_{2}(\tilde{k} x) /(\tilde{k} x)^{2}\right|:$

$$
F_{x} \approx \pi\left|p_{x}\right|^{2} \operatorname{Re}\left[\frac{(\tilde{k})^{3}\left(\tilde{k}_{1 z}\right)^{2} \tilde{k}_{2 z}}{k_{0}^{2}\left(\varepsilon_{1}-\varepsilon_{2}\right)} H_{1}^{(1)}(\tilde{k} x) \exp \left(2 i \tilde{k}_{1 z} z\right)\right] .
$$

The case of low losses is of special interest. Then, the final expression for the force can be reduced to

$$
F_{x}=\pi\left|p_{x}\right|^{2} \frac{\left(k^{*}\right)^{3}\left|k_{1 z}^{*}\right|^{2}\left|k_{2 z}^{*}\right|}{k_{0}^{2}\left(\varepsilon_{1}-\varepsilon_{2}\right)} Y_{1}\left(k^{*} r\right) \exp \left(-2 k_{1 z}^{*} z\right)
$$


where $Y_{1}(q)$ is the cylindrical Webber function. By expanding this expression around the zeros $q_{n}$ of the Webber function $Y_{1}(q) \approx-Y_{2}\left(q_{n}\right)\left(q-q_{n}\right)$, one can find the expression for the stiffness at the $n$th equilibrium position along the $x$ axis of the system [see Fig. 3(a)]:

$$
\kappa_{n} \approx \pi\left|p_{x}\right|^{2} \frac{(\tilde{k})^{3}\left|\tilde{k}_{1 z}\right|^{2}\left|\tilde{k}_{2 z}\right|}{k_{0}^{2}\left(\varepsilon_{1}-\varepsilon_{2}\right)} Y_{2}\left(q_{2 n-1}\right) \exp \left(-2\left|\tilde{k}_{1 z}\right| z\right) .
$$

Note that in the regime of SPP excitation without ohmic losses the SPP wave vector can be in the range from $k_{0}$ to $+\infty$ when $\varepsilon_{1}+\varepsilon_{2} \rightarrow 0$. With that, the expression Eq. (C11) goes to zero in both limiting cases,

$$
\kappa_{n} \underset{\tilde{k} \rightarrow 0, \infty}{\longrightarrow} 0
$$

which implies that the stiffness reaches its maximum at some particular wavelength. This wavelength can be defined for each given distance over substrate $z$. The maximal stiffness can be achieved close to SPP resonance when SPP wave vector equals $\tilde{k} \approx 3 / z$. Close to the frequency of SPP resonance when $\tilde{k} \rightarrow \infty$, the SPP becomes highly localized close to the interface $\left|\tilde{k}_{z}\right| \gg k_{0}$. Then, the maximal stiffness can be expressed as

$$
\kappa_{n} \sim\left|p_{x}\right|^{2}\left(\frac{3}{z}\right)^{6} \frac{1}{k_{0}^{2} \varepsilon_{1}} Y_{2}\left(q_{2 n+1}\right) \exp (-3) .
$$

\section{APPENDIX D: DYNAMICS SIMULATION}

We write Newton's law for the second particle,

$$
m \frac{d^{2}}{d t^{2}} \mathbf{r}_{2}=\mathbf{F}_{2}
$$

where $\mathbf{F}_{2}$ is given by Eq. (A1). One can rewrite it as

$$
\begin{aligned}
\frac{d^{2}}{d t^{2}} \mathbf{r}_{2}= & \frac{1}{2 m} \sum_{i} \operatorname{Re}\left\{p _ { 2 i } ^ { * } \nabla \left[E_{0 i}+\frac{k^{2}}{\varepsilon_{0}} \sum_{j} G_{s, i j}\left(\mathbf{r}_{2}, \mathbf{r}_{2}\right) p_{2 j}\right.\right. \\
& \left.\left.\left.+\frac{k^{2}}{\varepsilon_{0}} \sum_{j} G_{i j}\left(\mathbf{r}_{2}, \mathbf{r}_{1}\right) p_{1 j}\right]\right\}, \quad i, j=x, y, z, \quad \text { (D } 1\right)
\end{aligned}
$$

where $\widehat{G}=\widehat{G}_{0}+\widehat{G}_{s}$. In order to decrease the numerical error in the numerical simulations, we apply the following natural scaling:

$$
\begin{aligned}
\xi & =\frac{\mathbf{r}}{a}, \quad \tau=\frac{t}{T}, \quad \widetilde{\mathbf{E}}=\frac{\mathbf{E}}{E_{0}}, \quad \widetilde{\widetilde{G}}=a \widehat{G}, \\
\widetilde{\mathbf{k}} & =a \mathbf{k}, \quad \widetilde{\mathbf{p}}=\frac{\mathbf{p}}{4 \pi \varepsilon_{0} a^{3} E_{0}}, \quad \widetilde{\alpha}=\frac{\alpha}{4 \pi \varepsilon_{0} a^{3}}, \\
T & =\sqrt{\frac{m}{2 \pi \varepsilon_{0} a E_{0}^{2}}} .
\end{aligned}
$$

After such substitutions, we have

$$
\begin{aligned}
\frac{d^{2}}{d \tau^{2}} \xi_{2}= & \sum_{i} \operatorname{Re}\left\{\widetilde { p } _ { 2 i } ^ { * } \frac { \partial } { \partial \xi } \left[\widetilde{E}_{0 i}+4 \pi \widetilde{k}^{2} \sum_{j} \widetilde{G}_{i j}^{s}\left(\xi_{2}, \xi_{2}\right) \widetilde{p}_{2 j}\right.\right. \\
& \left.\left.+4 \pi \widetilde{k}^{2} \sum_{j} \widetilde{G}_{i j}\left(\xi_{2}, \xi_{1}\right) \widetilde{p}_{1 j}\right]\right\}
\end{aligned}
$$

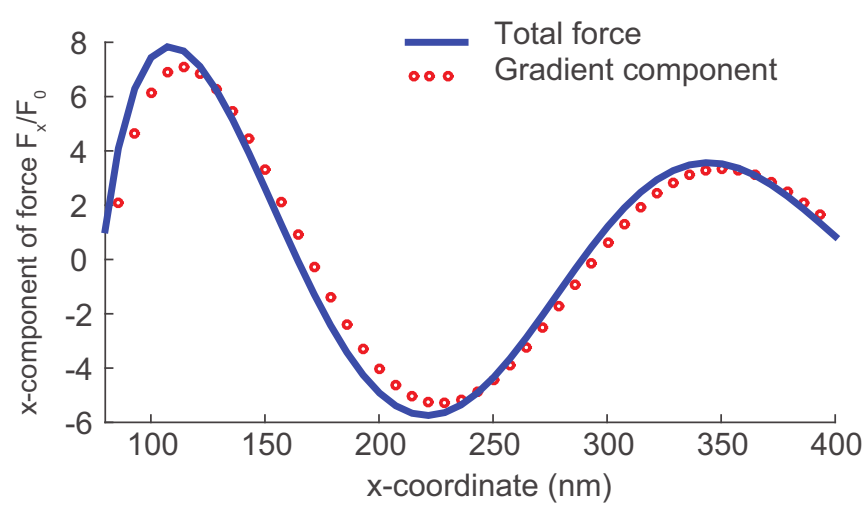

FIG. 7. The total force (blue solid line) and the conservative (red circles) components of the optical force are shown for different wavelengths. The parameters of the calculation are the same as in Fig. 2 of the main text.

We also include the viscosity of the environment by adding the damping factor $\gamma$,

$$
\frac{d^{2}}{d \tau^{2}} \xi_{2}=\widetilde{\mathbf{F}}_{2}\left(\xi_{1}, \boldsymbol{\xi}_{2}\right)-\gamma \frac{d}{d \tau} \xi_{2}
$$

where $\widetilde{\mathbf{F}}_{2}$ is given by the right-hand side of Eq. (D3). Expression (D4) was a target for the numerical simulation. As a good compromise between stability and computational complexity, the Runge-Kutta method of fourth order was applied. Since the motion along the $z$ axis is fixed, we have plane symmetry, which simplifies the force function to $\widetilde{\mathbf{F}}_{2}\left(\boldsymbol{\xi}_{1}, \boldsymbol{\xi}_{2}\right)=\widetilde{\mathbf{F}}_{2}$ $\left(\xi_{1}-\xi_{2}\right)$.

\section{APPENDIX E: CONSERVATIVE VS NONCONSERVATIVE FORCE COMPONENTS}

Here, we illustrate the contributions of conservative and nonconservative components of the optical force. The total optical force can be described as $\mathbf{F}=\frac{1}{4} \operatorname{Re}\left(\widehat{\alpha}_{\text {eff }}\right) \nabla\left|E_{0}\right|^{2}+$ $\frac{1}{2} \operatorname{Im}\left(\widehat{\alpha}_{\text {eff }}\right)\left|E_{0}\right|^{2} \nabla \varphi$, where $\varphi$ is the phase of the field. The first term corresponds to the gradient (conservative) force, which is proportional to a real part of particles' polarizability, while the second term corresponds to the scattering (nonconservative) force and is proportional to an imaginary part. By excluding the imaginary part of polarizability one can obtain the conservative force only $[43,46,47]$. The result is shown in Fig. 7. One can see that for the considered set of the parameters the conservative force strongly dominates over the nonconservative one, which is the difference between the total force and the gradient one.

\section{APPENDIX F: STIFFNESS OF THE OPTICAL TRAP FOR DIFFERENT PARTICLES' PARAMETERS}

The stiffness of the optical binding can be significantly influenced by the parameters of bounded particles. As can be seen from Eqs. (C11) and (2), the dielectric permittivity and the radius of the particles can change the value of the polarizability and stiffness. This is illustrated in Fig. 8(a), where the spectral stiffness of the trap is shown for different 

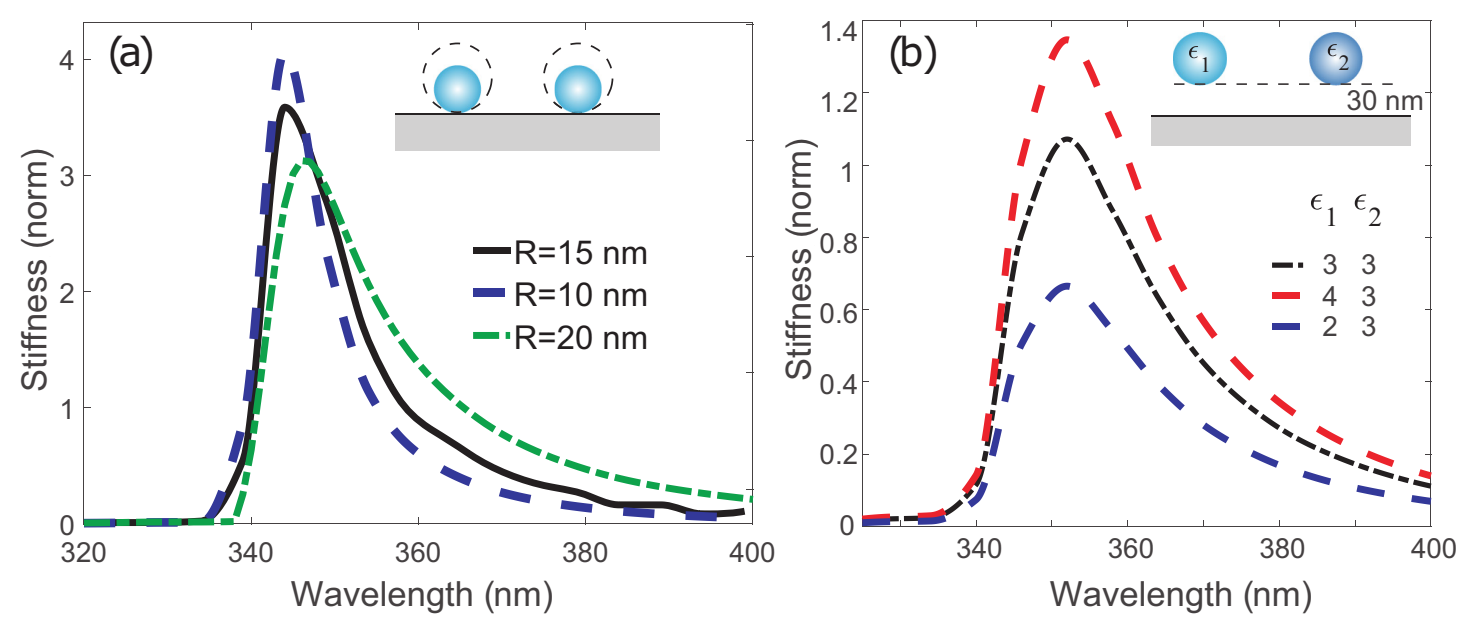

FIG. 8. (a) The stiffness $\kappa_{x}$ of the second equilibrium position in units of $\kappa_{0}=F_{0} / R$ as a function of the excitation wavelength. The spectra are shown for different radiuses $R$ of the nanoparticles. The distance from the surface is zero. (b) The stiffness spectra for nanoparticles made of different materials and $R=15 \mathrm{~nm}$.

nanoparticle sizes. We hold the distance between the surface of the particle and the metal interface constant, while varying the nanoparticle size as shown in the inset. The nanoparticle is described by a point dipole placed in the center of the sphere; thus, increasing the radius effectively increases the distance between the dipole and the surface, making the coupling between the dipole and SPP mode weaker. This provides the decrease of the stiffness with the nanoparticle radius increase. Actually, one may note that the absolute value of the stiffness will be increased in the end, as the normalization constant increases with the nanoparticle radius $\kappa_{0} \sim R^{2}$ due to the increase of polarizability of the particle. Similar behavior was observed in case of single particle near plasmonic substrate [33].
The case of nonidentical nanoparticles is of a special interest. We analyzed the stiffness of binding for two nanoparticles of different permittivities. The results are shown in Fig. 8(b), where the spectra of the stiffness parameter are shown for three values of the permittivities. We need to note that in the case of different nanoparticles the system loses symmetry and a constant force acting on the nanoparticle center of mass may appear. To avoid speculating on that, we fix the position of the first nanoparticle at the origin. Lower permittivity results in decrease of maximal stiffness as the intensity of excited SPPs is reduced due to the lower dipole moment, and coupling between the nanoparticles gets weaker. In contrast, the increase of the permittivity gives an increase of binding stiffness.
[1] O. M. Maragò, P. H. Jones, P. G. Gucciardi, G. Volpe, and A. C. Ferrari, Nat. Nanotechnol. 8, 807 (2013).

[2] I. Bloch, J. Dalibard, and S. Nascimbène, Nat. Phys. 8, 267 (2012).

[3] A. Ashkin, Phys. Rev. Lett. 24, 156 (1970).

[4] V. S. Letokhov, ZhETF Pisma Redaktsiiu. 7, 348 (1968).

[5] M. M. Burns, J. M. Fournier, and J. A. Golovchenko, Phys. Rev. Lett. 63, 1233 (1989).

[6] M. M. Burns, J. M. Fournier, and J. A. Golovchenko, Science (N.Y.) 249, 749 (1990).

[7] F. Depasse and J. M. Vigoureux, J. Phys. D 27, 914 (1994).

[8] P. C. Chaumet and M. Nieto-Vesperinas, Phys. Rev. B 64, 035422 (2001).

[9] J. Kesava, P. C. Chaumet, T. Langtry, and A. Rahmani, J. Nanophoton. 4, 041583 (2010).

[10] M. Mazilu, A. Rudhall, E. M. Wright, and K. Dholakia, J. Phys.: Condens. Matter 24, 464117 (2012).

[11] S. Sukhov, A. Shalin, D. Haefner, and A. Dogariu, Opt. Exp. 23, 247 (2015).

[12] R. W. Bowman and M. J. Padgett, Rep. Prog. Phys. Phys. Society (Great Britain) 76, 026401 (2013).
[13] M.-T. Wei, J. Ng, C. T. Chan, and H. D. Ou-Yang, Sci. Rep. 6, 38883 (2016).

[14] L. Chvatal, O. Brzobohaty, and P. Zemanek, Opt. Rev. 22, 157 (2015).

[15] V. Demergis and E. L. Florin, Nano Lett. 12, 5756 (2012).

[16] S. H. Simpson, P. Zemánek, O. M. Maragò, P. H. Jones, and S. Hanna, Nano Lett. 17, 3485 (2017).

[17] M. M. Salary and H. Mosallaei, Phys. Rev. B 94, 035410 (2016).

[18] C. Van Vlack, P. Yao, and S. Hughes, Phys. Rev. B 83, 245404 (2011).

[19] M. L. Juan, M. Righini, and R. Quidant, Nat. Photon. 5, 349 (2011).

[20] R. Quidant and C. Girard, Laser Photon. Rev. 2, 47 (2008).

[21] A. S. Shalin and S. V. Sukhov, Plasmonics 8, 625 (2013).

[22] A. S. Shalin, P. Ginzburg, A. A. Orlov, I. Iorsh, P. A. Belov, Y. S. Kivshar, and A. V. Zayats, Phys. Rev. B 91, 125426 (2015).

[23] A. P. Slobozhanyuk, P. Ginzburg, D. A. Powell, I. Iorsh, A. S. Shalin, P. Segovia, A. V. Krasavin, G. A. Wurtz, V. A. Podolskiy, P. A. Belov, and A. V. Zayats, Phys. Rev. B 92, 195127 (2015). 
[24] A. V. Chebykin, A. A. Orlov, A. S. Shalin, A. N. Poddubny, and P. A. Belov, Phys. Rev. B 91, 205126 (2015).

[25] A. Ivinskaya, N. Kostina, A. Proskurin, M. I. Petrov, A. A. Bogdanov, S. Sukhov, A. A. Krasavin, A. Karabchevsky, A. S. Shalin, and P. Ginzburg, ACS Photon. 5, 4371 (2018).

[26] A. A. Bogdanov, A. S. Shalin, and P. Ginzburg, Sci. Rep. 5, 15846 (2015).

[27] V. Garcés-Chávez, R. Quidant, P. J. Reece, G. Badenes, L. Torner, and K. Dholakia, Phys. Rev. B 73, 085417 (2006).

[28] G. Volpe, R. Quidant, G. Badenes, and D. Petrov, Phys. Rev. Lett. 96, 238101 (2006).

[29] M. Yuan, L. Cheng, P. Cao, X. Li, X. He, and X. Zhang, Plasmonics. 13, 427 (2018).

[30] Z. Yan, Y. Bao, U. Manna, R. A. Shah, and N. F. Scherer, Nano Lett. 14, 2436 (2014).

[31] C. Min, Z. Shen, J. Shen, Y. Zhang, H. Fang, G. Yuan, L. Du, S. Zhu, T. Lei, and X. Yuan, Nature Commun. 4, 2891 (2013).

[32] S. B. Wang and C. T. Chan, Nature Commun. 5, 3307 (2014).

[33] M. I. Petrov, S. V. Sukhov, A. A. Bogdanov, A. S. Shalin, and A. Dogariu, Laser Photon. Rev. 10, 116 (2016).

[34] A. Ivinskaya, M. I. Petrov, A. A. Bogdanov, I. Shishkin, P. Ginzburg, and A. S. Shalin, Light: Science \& Applications 6, e16258 (2017).

[35] S. Sukhov, V. Kajorndejnukul, R. R. Naraghi, and A. Dogariu, Nature Photon. 9, 809 (2015).
[36] F. J. Rodríguez-Fortuño, N. Engheta, A. Martínez, and A. V. Zayats, Nat. Commun. 6, 8799 (2015).

[37] S. Scheel, S. Y. Buhmann, C. Clausen, and P. Schneeweiss, Phys. Rev. A 92, 043819 (2015).

[38] F. J. Valdivia-Valero and M. Nieto-Vesperinas, Opt. Exp. 20, 13368 (2012).

[39] J. M. Auñón, F. J. Valdivia-Valero, and M. Nieto-Vesperinas, J. Opt. Soc. Am. A 31, 206 (2014).

[40] K. Dholakia and P. Zemánek, Rev. Modern Phys. 82, 1767 (2010).

[41] P. C. Chaumet and M. Nieto-Vesperinas, Opt. Lett. 25, 1065 (2000).

[42] P. B. Johnson and R. W. Christy, Phys. Rev. B 6, 4370 (1972).

[43] L. Novotny and B. Hecht, Principles of Nano-optics (Cambridge University Press, Cambridge, UK, 2012), p. 578.

[44] B. Khlebtsov, A. Melnikov, V. Zharov, and N. Khlebtsov, Nanotechnology 17, 1437 (2006).

[45] M. Abramowitz and I. A. Stegun, Handbook of Mathematical Functions: With Formulas, Graphs, and Mathematical Tables, Applied Mathematics Series (National Bureau of Standards, Washington, DC, 1964).

[46] A. S. Ang, S. V. Sukhov, A. Dogariu, and A. S. Shalin, Sci. Rep. 7, 41014 (2017).

[47] A. S. Ang, A. Karabchevsky, I. V. Minin, O. V. Minin, S. V. Sukhov, and A. S. Shalin, Sci. Rep. 8, 2029 (2018). 